Protocol design and ideas research exchange

\title{
Effects of Resistance Exercise with Instability on Neurocognitive Functions (REI STUDY): Study Protocol for a Proof-of-Concept Clinical Trial in Older Adults with Subjective Cognitive Complaints
}

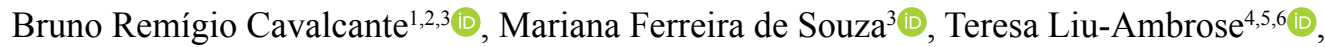 \\ David Behm ${ }^{\top}$, Ana Carolina Rodarti Pitangui ${ }^{2}$, Rodrigo Cappato de Araújo ${ }^{1,2}$ (1)
}

\begin{abstract}
${ }^{1}$ Universidade de Pernambuco, UPE, Associated Graduate Program in Physical Education UPE-UFPB, Recife, PE, Brazil; ${ }^{2}$ Universidade de Pernambuco, UPE, Laboratório de Pesquisa em Saúde e Desempenho Funcional, LABSED, Department of Physical Therapy, Petrolina, PE, Brazil, ${ }^{3}$ Universidade Federal do Vale do São Francisco, UNIVASF, Clinical Exercise Lab, LABEC, Department of Physical Education, Petrolina, PE, Brazil; ${ }^{4}$ University of British Columbia, UBC, Department of Physical Therapy, Vancouver, Canada; ${ }^{5}$ Djavad Mowafaghian Centre for Brain Health, Vancouver Coastal Health Research Institute, Vancouver, Canada. ${ }^{6}$ Centre for Hip Health and Mobility, Vancouver Coastal Health Research Institute, Vancouver, Canada; ${ }^{7}$ School of Human Kinetics and Recreation, Memorial University of Newfoundland St. John's, NL, Canada.
\end{abstract}

\begin{abstract}
Aim: Resistance exercise (RE) is a widely accepted strategy to counteract the effects of aging and chronic diseases on health-related outcomes. Studies have been suggested that RE improves brain and cognitive health. RE with unstable devices (REI) is a neuromotor complexity strategy with the potential to improve strength, power, and balance in onder adults, however, their effects on neurocognitive function in individuals at risk of cognitive decline are poorly understood. Thus, we describe the REI study protocol, a randomized clinical trial design to assess the effects of 12-weeks of REI and RE on neurocognitive functions in older adults with subjective cognitive complaints. Methods: The sample will consist of individuals (aged 65 or older) with subjective cognitive complaints, who will be randomized in one of three experimental groups: control, RE or REI. Participants of the RE group will receive 12-weeks of progressive resistance training (seven exercises structured into 3 sets of 10-15 repetitions maximum, three times per week). Participants of the REI group will receive the same RE protocol performed on unstable devices that promote progressive instability. The control group received a health education program. The primary outcome is a composite score of neurocognitive functions. Secondary outcomes include each separately neurocognitive domain, functional performance, balance, falls-related parameters, cardiovascular and mood variables. The study results will provide information to design a future and larger preventive trial on populations at risk of cognitive decline and dementia, which is imperative in public health terms. Trial registration: Brazilian registry of clinical trials number RBR-4kqs 22.
\end{abstract}

Keywords: resistance training; instability; aging; cognitive impairment; study protocol.

\section{Background}

Dementia is a neurodegeneration syndrome associated with deterioration of cognitive health and functional activities. It is estimated that 35.6 million people lived with dementia in 2010 and projections for 2050 indicate that approximately 115.4 million people will be affected by this clinical condition ${ }^{1}$. Therefore, reducing the dementia burden has been a public health challenge.

Subjective cognitive decline is an established dementia risk factor $^{2}$. Pharmacological strategies to ameliorate the cognitive impairment have demonstrated low efficacy in clinical populations with cognitive decline ${ }^{3}$, whereas non-pharmacological strategies based on behavioral changes including exercise $\mathrm{e}^{4,5}$ have been shown to be a promising alternative to attenuate the deleterious effects on physical and cognitive health-related outcomes in people at risk of cognitive impairment.
Progressive resistance exercise (RE) programs have been widely recommended for the geriatric population due to its health benefits including gains and maintenance of strength, and muscle mass, bone components, functional performance, cardiometabolic health and fall prevention ${ }^{6}$. Evidence has also suggested that progressive RE programs can provide benefits in neurocognitive and brain function in healthy ${ }^{7-9}$ individuals and those with cognitive impairment ${ }^{10}$. For instance, in the SMART study, Fiatarone et al. ${ }^{10}$ found improved cognition after a progressive RE in older adults with mild cognitive impairment, which might represent a potential adjuvant non-pharmacological strategy for the prevention and treatment of neurocognitive disorders.

Additionally, studies have highlighted activities that require elevated neuromotor complexity ${ }^{11-14}$ are associated with better clinical and health indicators, including cognitive outcomes. In this line, RT associated with unstable devices 
(as called Resistance Exercise with Instability - REI) is considered a strategy to promote greater neuromotor complexity, because it requires an elevated neuromotor and cognitive demand. A meta-analysis ${ }^{15}$ showed that REI protocols are effective to promote gains in strength, power and balance of older adults. To our best knowledge, only one study ${ }^{14}$ tested the effects of REI on neurocognitive function. In this study, Silva-Batista et $a 1^{14}$. showed significant improvements on Montreal Cognitive Assessment (MoCA) scores after 12-weeks of REI (mean difference $=+6$ points) in patients with Parkinson Disease compared with control group. Moreover, the proportion of patients classified with probable cognitive impairment (cut-off point $<26$ ) reduced by approximately $80 \%$, which favors the argumentation of this exercise strategy can be potentiated RT-induced gains in individuals with cognitive impairment.

Considering that cognitive and functional declines during the aging process may be potentiated in the older adults cognitively impaired ${ }^{16}$ and associated with poor health-related outcomes, it is postulated that the use of REI represents a valuable way to prevent and even attenuate the deleterious effects on cognitive health in this subgroup. Thus, our aim will be to assess the effects of 12 -weeks of REI and RE on neurocognitive functions in elderly with subjective cognitive complaints. Our hypothesis is that, compared with control group, REI significantly improve neurocognitive functions.

\section{Methods}

\section{Experimental design}

This study will involve a proof-of-concept randomized clinical trial with parallel groups. The study will have a total duration of 12-weeks with two evaluation periods. The baseline procedures will involve: 1) pre-participation screening; 2) evaluation of primary and secondary outcomes. Subsequently, participants will be assigned to perform, on a random basis, REI or RE or health educational, stretching and relaxation activities. After 12-weeks the evaluations of the outcomes will be repeated. The experimental design and is presented in Figure 1.

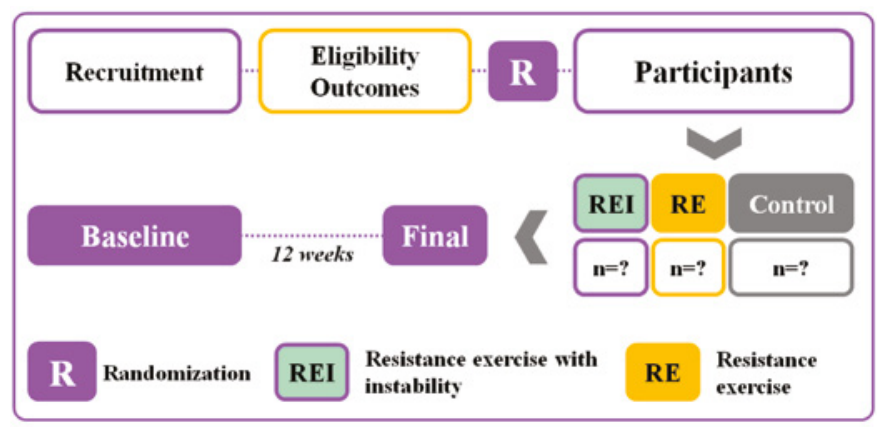

Figure 1. Experimental design and timeline of REI study.

\section{Participants, eligibility criteria e recruitment}

Community-dwelling older adults with subjective cognitive complaints (i.e. memory and/or attentional complaints) will participate of the study. The eligibility criteria will be: i) aged 65 and older; ii) physically inactive (no structured exercise classes in the last 3 months); iii) MoCA score lower than 26 points; iv) absence of clinical conditions or unstable disease that contraindicates supervised exercise based on medical judgment; v) no previously diagnosed cerebrovascular, neurological or psychiatric disease; and vi) have sufficient visual and hearing capacity to complete cognitive assessment protocol.

Study participants will be recruited between 2018 July and August through local advertising (TV \& radio, newspaper and business ads), social media, email invitations, direct contact with participants from previous studies, health services, community programs for the elderly and verbal communication.

\section{Ethics and Consent to Participate}

The study will be conducted under the Declaration of Helsinki principles and it was approved by the Ethics and Research Committee of the University of Pernambuco (CAAE protocol: 81016817.7.0000.5207). All participants will sign a written informed consent before baseline assessments. All stages of this study are in accordance with the recommendations of the Standard Protocol Items: Recommendations for Interventional Trials (SPIRIT).

\section{Randomization and blinding}

The randomization of participants will occur at the end of the baseline assessments in a 1:1:1 fashion through a sequence of randomly permuted blocks, stratified by sex and age, which will be generated by an independent researcher.

Given the nature of the interventions studied, therapists and patients, double blinding will be not possible. However, the evaluator and the statistician will not know to which group the subjects evaluated will belong. All participants will be instructed not to disclose what type of treatment they were receiving.

\section{Sample size calculation}

This is the first study to investigate the effects of REI on the neurocognitive functions of the elderly with subjective cognitive complaints, which limits a formal analysis for calculating the sample size. However, based on the results of different metaanalyzes $^{17,18}$, we used the following criteria for sample size of the study: effect size $=0.20$, alpha $=5 \%$; power $=80 \%$. From these estimates, a minimum number of 66 individuals (22 per group) will be recruited. Anticipating eventual losses and withdrawals in intervention groups, the sample will be inflated by $15 \%$, totaling 25 individuals per study arm $(n=75)$. 


\section{Intervention groups}

Health education control group - Participants from the control group will hold a weekly group meeting in order to provide health education activities including lectures on prevention and treatment of chronic diseases, discussions on the importance of maintaining a healthy lifestyle, as well as stretching and relaxation classes. This model of a control group is based on previous studies ${ }^{17}$ and has been recommended as a strategy to control the socializing effect of exercise-based interventions.

Resistance exercise group - The RE program will be performed over 12 weeks (3 times per week) on alternate days. The training program will consist of 7 exercises for the main muscle groups (Squat, dumbbell press, leg press, paddled on the pulley, pelvic elevation, plantar flexion, and abdominal exercises) performed at the same time of day. The prescription will involve three fixed sets and repetitions ranging from $10-15$ repetition maximum (RM), with the technique of movement performed correctly. With abdominal exercises, the prescription will involve the execution of three sets of 15-30 repetitions or isometric contractions ranging from 10-30 seconds. The recovery interval between the sets, in each exercise, will be from 60 to 90 seconds, and between exercises from two to three minutes. The training progression and adjustment of training loads will be performed according to the American College of Sports Medicine recommendations ${ }^{19}$.

Resistance exercise with instability group - The REI group will perform the same training program as the RE group, however, instability equipment (i.e. BOSU ${ }^{\circledR}$ ball, balance disc, and physio ball) will be included. During the intervention, the degree of postural instability in the exercises will progress as the individual improves his balance and/or muscle strength quickly ${ }^{20}$. The Table 1 shows a summary of the REI protocol.

Table 1. REI training protocol.

\begin{tabular}{|c|c|c|c|c|}
\hline Exercises & Weeks & Devices & Description & Training periodization \\
\hline All & $1-4$ & Absent & RE group protocol & \multirow{8}{*}{$\begin{array}{l}\text { Abdominal: } \\
15-30 \text { reps }\end{array}$} \\
\hline Squat & \multirow{7}{*}{$5-8$} & $\begin{array}{l}\text { Balance disc } \\
\text { Physioball }\end{array}$ & $\begin{array}{l}\text { Balance disc between feet and ground; Physioball } \\
\text { between back and wall }\end{array}$ & \\
\hline Bench press* & & Balance disc & Head, scapular waist and hip on Balance disc & \\
\hline Leg press & & Balance disc & Feet supported on Balance disc & \\
\hline Paddling & & Physioball & Sitting on the Physioball and feet on the floor & \\
\hline Pelvic elevation & & Balance disc & Bilateral feet supported on Balance disc & \\
\hline Plantar flexion and dorsiflexion & & & Standing plantar flexion and dorsiflexion & \\
\hline Abdominal & & & Abdominal plus plank frontal exercise & \\
\hline Squat & \multirow{7}{*}{$9-12$} & $\begin{array}{l}\text { BOSU }^{\circledR} \\
\text { Physioball } \\
\end{array}$ & $\begin{array}{l}\text { BOSU }^{\circledR} \text { between feet and ground; Physioball between } \\
\text { back and wall }\end{array}$ & \multirow{7}{*}{$\begin{array}{c}3 \text { sets } \\
10-15 \text { reps } \\
\Delta \mathrm{t} \text { sets: } 60 \mathrm{~s} \\
\Delta \mathrm{t} \text { exercises: } 120 \mathrm{~s} \\
\text { Abdominal: } \\
15-30 \text { reps and } 10-30 \mathrm{~s}\end{array}$} \\
\hline Bench press* & & Balance disc & Head, scapular waist on Balance disc & \\
\hline Leg press & & Balance disc & BOSU $^{\circledR}$ between the feet and the machine & \\
\hline Paddling & & $\begin{array}{l}\text { Physioball } \\
\text { Balance disc }\end{array}$ & $\begin{array}{l}\text { Sitting on the Physioball and Balance discs between } \\
\text { the feet and the floor }\end{array}$ & \\
\hline Pelvic elevation & & Balance disc & Unilateral feet supported on Balance disc & \\
\hline Plantar flexion and dorsiflexion & & & Standing plantar flexion and dorsiflexion & \\
\hline Abdominal & & Physioball & Abdominal plus plank frontal exercise & \\
\hline
\end{tabular}

*Dumbbell assistance.

\section{Adverse events monitoring}

The adverse events monitoring will be carried out monthly and obtained through face-to-face or telephone interview. The presence of adverse events will include worsening of health status or the appearance of musculoskeletal and cardiometabolic problems related to the intervention protocol.

\section{Pre-participation screening}

Demographic data, medical history, medication and supplement use (i.e. vitamin D), anthropometric measurements will be obtained. Instrumental and basic activities of daily living will be assessed using the self-report Lawton-Brody ${ }^{21}$ and Katz ${ }^{22}$ scales, respectively. The individuals with probable cognitive impairment will be assessed by the Brazilian version of the $\mathrm{MoCA}^{23}$ (scores $<26$ points on a score ranging from 0 to 30 ).

\section{Outcomes}

The table 2 summarizes the primary and secondary outcomes of the REI study. 
Table 2. REI study outcomes summary.

\begin{tabular}{|c|c|c|c|}
\hline Outcomes & Methods & Baseline & Post-12 weeks \\
\hline \multicolumn{4}{|l|}{ Primary } \\
\hline Neurocognitive functions & Composite score & $\bullet$ & • \\
\hline \multicolumn{4}{|l|}{ Secondary } \\
\hline \multirow[t]{8}{*}{ Neurocognitive domains } & Montreal Cognitive Assessment & • & • \\
\hline & Stroop task & $\bullet$ & • \\
\hline & Trail Making Test Part A \& B & $\bullet$ & $\bullet$ \\
\hline & Digit Span Backward \& Foward & $\bullet$ & $\bullet$ \\
\hline & Digit Symbol Coding Test & • & • \\
\hline & Verbal fluency & • & • \\
\hline & Time up \& go with cognitive demand & • & • \\
\hline & Logical memory & $\bullet$ & $\bullet$ \\
\hline \multirow[t]{4}{*}{ Functional performance } & Muscle strength & $\bullet$ & • \\
\hline & Short Physical Performance Battery & $\bullet$ & • \\
\hline & Six-minute walking test & • & • \\
\hline & Time up \& go without cognitive demand & $\bullet$ & $\bullet$ \\
\hline Balance & Force plate & $\bullet$ & $\bullet$ \\
\hline \multirow[t]{2}{*}{ Falls-related parameters } & Fall history & $\bullet$ & • \\
\hline & Fear of fall & • & • \\
\hline \multirow[t]{2}{*}{ Cardiovascular parameters } & Blood pressure & $\bullet$ & $\bullet$ \\
\hline & Cardiac autonomic modulation & • & • \\
\hline Mood & Depression symptoms & $\bullet$ & $\bullet$ \\
\hline
\end{tabular}

\section{Primary outcome}

\section{The composite score of neurocognitive functions}

The study will evaluate different neurocognitive domains through validated neuropsychological tests including: MoCA, Stroop Color Task, Trail Making Test Part A \& B, Digit Span Backward \& Forward, Digit Symbol Coding Test, Time up \& go with cognitive demand, Verbal fluency (animal and naming), and Logical memory (immediate and delayed recall). To evaluate the primary outcome, we will use a composite score of these neurocognitive functions from the sum of the data reduction obtained in each neuropsychological test for z-values. This strategy has been widely used in clinical studies that analyzed the effects of therapeutic interventions on the cognitive health of elderly people at risk of dementia ${ }^{24,25}$.

\section{Secondary outcomes}

\section{Global neurocognitive function domain}

The global cognitive function will be assessed by the Brazilian version of the $\mathrm{MoCA}^{23}$. The MoCA is a cognitive screening tool composed of 12 individual tasks, which enables assessment of eight different cognitive tasks. The average completion time for the MoCA is approximately 10 minutes and its score ranged from 0 to 30 points with a higher score of reflecting better cognition.

\section{Executive function, processing speed, and attention} domains

Stroop color task - The selective attention and conflict resolution will be carried out by a computerized Stroop color task $^{26}$. This test version consists of three phases: the first (congruent) and second (neutral) phases are control conditions (i.e., indicate the ink color of a rectangle; indicate the color word that appears in white ink), and the last phase includes the incongruent trials (i.e., indicate the ink color of color words). Selective attention and conflict resolution will be calculated as the mean difference in reaction time between incongruent and neutral phase responses.

Trail making test part $A \& B$ - The trail making test (TMT) $)^{27}$ consists of two parts ( $\mathrm{A}$ and $\mathrm{B}$ ) where the time required to complete each part is used as a measure of executive functioning and processing speed. In part A, the participant must draw a line that connects consecutive numbers from 1 to 25 . In part $\mathrm{B}$, the participant must draw a line that connects numbers and letters alternately and in ascending order.

Digit span forward \& backward - The evaluation of attention and working memory will be performed by testing the digit span forward \& backward ${ }^{28}$. Both consist of generating sequences of random numbers that begin with three digits and gradually increases to the maximum of nine digits. In the forward version, the participant task is to repeat each sequence in the same order (direct), whereas in the backward version, the task is to repeat each sequence in reverse order. The final score is based on the number of successful sequences, 
ranging from 0 to 14 (higher scores indicate better attention and working memory).

Digit symbol coding test - In this test, participants will receive a paper with a sequence of numbers (1 to 9) and corresponding symbols (at the top) and a random sequence of numbers and empty boxes to fill (at the bottom). The test consists of filling in the largest number of empty boxes with a symbol corresponding to each number. The time to perform the test is 120 seconds and the score is determined by the number of correctly completed number symbol matches.

\section{Language domain}

Semantic and phonological verbal fluency - The language domain will be evaluated by the semantic and phonological verbal fluency test ${ }^{29}$. This test allows to evaluating the number of words spontaneously evoked orally for one minute (by category). In the semantic category, the participants will be requested to speak the greatest number of animal names, while in the phonological category, the greatest number of words beginning with the letters F, A and S.

\section{Memory domain}

Logical memory - The memory domain will be evaluated from the immediate and delayed logical memory of the Wechsler Memory Scale (WMS-IV) ${ }^{30}$. Briefly, two different stories are read once to the participant, and later it is asked to verbally evoke any information that is remembered. The evaluator collects the amount of recall and thematic units (immediate recall). After an interval of 30 minutes, the participant is asked to describe any information remembered from the first and second story. In the absence of recall, a standard tip is made available. Finally, the scores of the recall and thematic units are again recorded (delayed recall).

\section{Dynamic, isometric and isokinetic strength}

The estimation of dynamic muscle strength will be assessed by a maximal repetition test (1-RM) for the lower limbs (leg press exercise $)^{31}$. Three sessions will be held at the same time of day with a 48-hour interval between sessions. The load recorded as 1-RM will be one in which it is possible to perform a single maximal voluntary action in the concentric and eccentric phases. A trained evaluator will conduct the evaluations, using consistent and standardized recommendations.

The maximum voluntary isometric contraction (MVIC) will be obtained with a hand-held dynamometer adjustable and calibrated with a scale of zero to $100 \mathrm{kgf}$, following the previously described procedures and recommendations $\mathrm{s}^{32}$.

The assessment of isokinetic quadriceps strength will be measured using an isokinetic dynamometer (Kin-Com Model $125 \mathrm{E}$, version 3.2; Chattanooga, $\mathrm{TN})^{33}$. The maximum quadriceps strength will be defined as the peak torque produced in two sets of five consecutive measures of concentric force on the knee extension (both legs) using a protocol with an angular velocity of 60 degrees per second adopting an interval of two to five minutes between attempts. To avoid compensatory movements during the evaluation, belts around the trunk and pelvis will be attached to the seat as described in the manufacturer's recommendations.

\section{Functional performance and balance}

Six-minute walking test - Briefly, the test ${ }^{34}$ consists of a walk along a 30-meter corridor where the participants will be encouraged to "walk at their usual pace for six-minutes and cover as much ground as possible" and rest, if and when necessary. At the end of each minute, participants received feedback on the elapsed time and standardized encouragement in the form of statements will be utilized by the same researcher. Total walking distance will be defined as the maximum distance completed at the end of six minutes.

Short physical performance battery - The test ${ }^{35}$ is composed of five tests that allow to evaluating the balance, gait, strength/ endurance of lower limbs. The total score obtained by the sum of the scores of each test allows values between zero and 12 points, with zero and 12 points reflecting the worst and best functional performance, respectively.

Time up \& go without and with cognitive demand - The time up \& go36 measures the time the participant needs to get up from a chair, walk to a line that will be 3 meters from the ground, walk around it (180 degrees) and return and sit back in the chair. The time up $\&$ go with cognitive demand ${ }^{37}$ will be performed using the same procedures adopted in the previous protocol, but the participants will be asked to perform a cognitive task (i.e. a random subtraction operation).

Balance - Static balance evaluation will be performed using a force platform ${ }^{38}$. The participants will remain statically on the platform in the supports: bipodal, semi-tandem, and unipodal dominant side, all performed with eyes open and closed. Each exam will be done three consecutive times, in a randomized manner. In the present study, the amplitude data of the displacement of the center of pressure $(\mathrm{CoP})$ in the anteroposterior, medial-medial direction and total mean velocity of CoP displacement will be analyzed.

\section{Fall related-parameters}

The history and incidence of falls will be evaluated by means of self-report (history of fall in the last year) and face-to-face or telephone interviews that will be performed twice a month during the intervention. In accordance with a previous consensus, fall as an unexpected event in which participants came to rest on the ground, floor, or lower level ${ }^{39}$.

To evaluate the concerns about falling, the validated and translated version of Falls Efficacy Scale-International ${ }^{40}$ will be used. This instrument encompasses questions about the concerns about falling when performing 16 activities with scores ranging from one to four. The total score can range from 16 (without concern) to 64 (extreme concern). 


\section{Cardiovascular parameters}

Blood pressure - All procedures for the measurement of blood pressure (BP) will follow the recommendations of the VII Brazilian Guidelines on Hypertension ${ }^{41}$. The BP measurement will be performed in triplicate through a digital monitor (HEM742INT, Omron, Brazil).

Cardiac autonomic modulation - Cardiac autonomic modulation will be assessed by heart rate variability (HRV) analysis $^{42}$. Participants will remain at rest for 15 minutes and during the same period, the R-R intervals will be continuously recorded by means of a validated heart rate monitor (Polar V800). The analysis of the cardiac autonomic modulation indicators will be performed through the Kubios HRV program, and the information will be interpreted from the time and frequency domains.
Mood

The symptoms of depression will be assessed through the short version of the Geriatric Depression Scale ${ }^{43}$. The presence of depressive symptoms will be defined from the five or more symptoms in the 15 items or the equivalent for those participants who respond from 10 to 14 responses.

\section{Time schedule}

The provisional schedule of the REI study is described in Table 3. The study will have a total estimated duration of 2 years.

Table 3. Provisional calendar for the REI study.

\begin{tabular}{|c|c|c|c|c|c|}
\hline \multirow{2}{*}{ Funding year } & \multirow{2}{*}{$\begin{array}{c}2017 \\
\text { Sept-Dec }\end{array}$} & \multicolumn{2}{|c|}{2018} & \multicolumn{2}{|c|}{2019} \\
\hline & & Feb-May & Jun-Dec & Feb-May & Jun-Dec \\
\hline \multicolumn{6}{|l|}{ Activity } \\
\hline Study setting up & $\bullet$ & $\bullet$ & & & \\
\hline Research team training & & $\bullet$ & $\bullet$ & & \\
\hline Project disclosure & & $\bullet$ & $\bullet$ & & \\
\hline Volunteers recruitment & & & $\bullet$ & & \\
\hline Baseline assessments & & & $\bullet$ & & \\
\hline Interventions & & & $\bullet$ & & \\
\hline Close-out assessments & & & • & & \\
\hline Data analysis & & & • & $\bullet$ & $\bullet$ \\
\hline Publications & & & $\bullet$ & $\bullet$ & $\bullet$ \\
\hline
\end{tabular}

\section{Statistical analysis}

First, exploratory analyses will be performed to verify the distribution of variables, identification of outliers, missing data, and asymmetries. The effects of the intervention on outcomes of interest will be analyzed by mixed generalized linear models, considering the groups (REI, RE, and control) and time (baseline and post-12 weeks) as fixed factors and the individual as a random factor. When differences are detected, the Sidak post hoc will be used for multiple comparisons. The intention-to-treat analysis will be applied.

All statistical procedures will be performed by an independent researcher (blinded for interventions) using SPSS software version 22 (IBM SPSS Corporation, New York, USA). The significance level adopted will be $5 \%$.

\section{Discussion}

The REI study is a proof-of-concept randomized clinical trial elaborated to analyze and compared the efficacy of 12-weeks REI and RE on neurocognitive outcomes of older adults with subjective cognitive complaints.
RE programs have benefits not only in terms of strength and body composition ${ }^{6}$ but also for cognitive and brain health in both healthy and cognitive impaired older adults individuals ${ }^{5,17}$, and therefore, their use should be recommended to counteract the deleterious effects of aging as well as the chronic diseases that are highly prevalent in this stage of lifespan.

More recently, studies that combined RE with unstable devices protocols have been used in preventive ${ }^{44}$ and therapeutic contexts $^{12}$, as a strategy of elevated neuromotor complexity with potential to promote positive gains in the health-related outcomes. A recent meta-analysis ${ }^{15}$ demonstrated that the REI improves muscle strength, power and balance of the older adults ${ }^{45}$, whereas, in clinical populations, this exercise mode strategy was able to improve the cognitive functions of patients with Parkinson's disease ${ }^{14}$. In this line, it is reasonable to hypothesize that REI is an approach that will also promote beneficial changes in the neurocognitive function in subjective cognitive complaints people.

The results obtained from the REI study will aid to the understanding of the effects of RE and REI on the neurocognitive functions of the older adults with subjective cognitive complaints, a population at risk for decline in the 
cognitive and functional phenotypes. In addition, the REI study will provide information for further larger clinical trials.

\section{Availability of data and materials}

Data and materials sharing are not applicable to this study protocol article as no datasets were analyzed during the current article. However, in agreement with the current tendencies for the sharing and dissemination in science, the data will be available to outside investigators at the end of the study and the main findings will be published in peer-reviewed Journals as well as presented at national and international conferences. Additionally, the results will be released to all participants.

\section{References}

1. Livingston G, Sommerlad A, Orgeta V, Costafreda SG, Huntley J, Ames D, Ballard C, Banerjee S, Burns A, Cohen-Mansfield $\mathrm{J}$ et al: Dementia prevention, intervention, and care. Lancet 2017, 390(10113):2673-2734.

2. Rabin LA, Smart CM, Amariglio RE: Subjective Cognitive Decline in Preclinical Alzheimer's Disease. Annu Rev Clin Psychol 2017, 13:369-396.

3. Petersen RC, Lopez O, Armstrong MJ, Getchius TSD, Ganguli M, Gloss D, Gronseth GS, Marson D, Pringsheim T, Day GS et al: Practice guideline update summary: Mild cognitive impairment: Report of the Guideline Development, Dissemination, and Implementation Subcommittee of the American Academy of Neurology. Neurology 2018, 90(3):126-135.

4. Jackson PA, Pialoux V, Corbett D, Drogos L, Erickson KI, Eskes GA, Poulin MJ: Promoting brain health through exercise and diet in older adults: a physiological perspective. J Physiol 2016, 594(16):4485-4498.

5. Saez de Asteasu ML, Martinez-Velilla N, Zambom-Ferraresi F, Casas-Herrero A, Izquierdo M: Role of physical exercise on cognitive function in healthy older adults: A systematic review of randomized clinical trials. Ageing Res Rev 2017, 37:117-134.

6. Westcott WL: Resistance training is medicine: effects of strength training on health. Curr Sports Med Rep 2012, 11(4):209-216.

7. Liu-Ambrose T, Nagamatsu LS, Graf P, Beattie BL, Ashe MC, Handy TC: Resistance training and executive functions: a 12-month randomized controlled trial. Arch Intern Med 2010, 170(2):170-178.

8. Liu-Ambrose T, Nagamatsu LS, Voss MW, Khan KM, Handy TC: Resistance training and functional plasticity of the aging brain: a 12-month randomized controlled trial. Neurobiol Aging 2012, 33(8):1690-1698.

9. Tsai CL, Wang CH, Pan CY, Chen FC: The effects of long-term resistance exercise on the relationship between neurocognitive performance and GH, IGF-1, and homocysteine levels in the elderly. Front Behav Neurosci 2015, 9:23.
10. Fiatarone Singh MA, Gates N, Saigal N, Wilson GC, Meiklejohn J, Brodaty H, Wen W, Singh N, Baune BT, Suo C et al: The Study of Mental and Resistance Training (SMART) study-resistance training and/or cognitive training in mild cognitive impairment: a randomized, double-blind, double-sham controlled trial. J Am Med Dir Assoc 2014, 15(12):873-880.

11. Carey JR, Bhatt E, Nagpal A: Neuroplasticity promoted by task complexity. Exerc Sport Sci Rev 2005, 33(1):24-31.

12. Behm DG, Drinkwater EJ, Willardson JM, Cowley PM: Canadian Society for Exercise Physiology position stand: The use of instability to train the core in athletic and nonathletic conditioning. Appl Physiol Nutr Metab 2010, 35(1):109-112.

13. Eckardt N: Lower-extremity resistance training on unstable surfaces improves proxies of muscle strength, power and balance in healthy older adults: a randomised control trial. BMC Geriatr 2016, 16(1):191.

14. Silva-Batista C, Corcos DM, Roschel H, Kanegusuku H, Gobbi LT, Piemonte ME, Mattos EC, MT DEM, Forjaz CL, Tricoli V et al: Resistance Training with Instability for Patients with Parkinson's Disease. Med Sci Sports Exerc 2016, 48(9):1678-1687.

15. Behm DG, Muehlbauer T, Kibele A, Granacher U: Effects of Strength Training Using Unstable Surfaces on Strength, Power and Balance Performance Across the Lifespan: A Systematic Review and Metaanalysis. Sports Med 2015, 45(12):1645-1669.

16. Montero-Odasso M, Speechley M: Falls in Cognitively Impaired Older Adults: Implications for Risk Assessment And Prevention. J Am Geriatr Soc 2018, 66(2):367-375.

17. Northey JM, Cherbuin N: Exercise interventions for cognitive function in adults older than 50: a systematic review with meta-analysis. $\mathrm{Br}$ J Sports Med 2018, 52(3):154-160.

18. Gates N, Fiatarone Singh MA, Sachdev PS, Valenzuela M: The effect of exercise training on cognitive function in older adults with mild cognitive impairment: a meta-analysis of randomized controlled trials. Am J Geriatr Psychiatry 2013, 21(11):1086-1097.

19. American College of Sports Medicine position stand. Progression models in resistance training for healthy adults. Med Sci Sports Exerc 2009, 41(3):687-708.

20. Sherrington C, Michaleff ZA, Fairhall N, Paul SS, Tiedemann A, Whitney J, Cumming RG, Herbert RD, Close JCT, Lord SR: Exercise to prevent falls in older adults: an updated systematic review and meta-analysis. Br J Sports Med 2017, 51(24):1750-1758.

21. Lawton MP, Brody EM: Assessment of older people: self-maintaining and instrumental activities of daily living. Gerontologist 1969, 9(3):179-186.

22. Katz S: Assessing self-maintenance: activities of daily living, mobility, and instrumental activities of daily living. J Am Geriatr Soc 1983, 31(12):721-727.

23. Memoria CM, Yassuda MS, Nakano EY, Forlenza OV: Brief screening for mild cognitive impairment: validation of the Brazilian version of the Montreal cognitive assessment. Int J Geriatr Psychiatry 2013, 28(1):34-40.

24. Ngandu T, Lehtisalo J, Solomon A, Levalahti E, Ahtiluoto S, Antikainen R, Backman L, Hanninen T, Jula A, Laatikainen T et al: A 2 year multidomain intervention of diet, exercise, cognitive training, and vascular risk monitoring versus control to prevent cognitive decline in at-risk elderly people (FINGER): a randomised controlled trial. Lancet 2015, 385(9984):2255-2263. 
25. Andrieu S, Guyonnet S, Coley N, Cantet C, Bonnefoy M, Bordes S, Bories L, Cufi MN, Dantoine T, Dartigues JF et al: Effect of long-term omega 3 polyunsaturated fatty acid supplementation with or without multidomain intervention on cognitive function in elderly adults with memory complaints (MAPT): a randomised, placebo-controlled trial. Lancet Neurol 2017, 16(5):377-389.

26. da Silva WQA, Fontes EB, Forti RM, Lima ZL, Machado D, Deslandes AC, Hussey E, Ward N, Mesquita RC, Okano AH et al: Affect during incremental exercise: The role of inhibitory cognition, autonomic cardiac function, and cerebral oxygenation. PLoS One 2017, 12(11):e0186926.

27. Carone DA: E. Strauss, E. M. S. Sherman \& O. Spreen, A Compendium of Neuropsychological Tests: Administration, Norms, and Commentary. Applied Neuropsychology 2007, 14(1):62--63.

28. Richardson JT: Measures of short-term memory: a historical review. Cortex 2007, 43(5):635-650.

29. Whiteside DM, Kealey T, Semla M, Luu H, Rice L, Basso MR, Roper B: Verbal Fluency: Language or Executive Function Measure? Appl Neuropsychol Adult 2016, 23(1):29-34.

30. Wechsler D: Wechsler Memory Scale (WMS-IV) - Fourth Edition: Pearson; 2009.

31. Souza MF, Tomeleri CM, Ribeiro AS, Schoenfeld BJ, Silva AM, Sardinha LB, Cyrino ES: Effect of resistance training on phase angle in older women: A randomized controlled trial. Scand J Med Sci Sports 2017, 27(11):1308-1316.

32. Farah B, Correia M, Rodrigues S, Cavalcante B, Ritti-Dias R: Reliability of handgrip maximal voluntary contraction in hypertensive adults. Rev Bras Ativ Fis e Saúde 2014, $19(5$ ):590-596.

33. Fabbri E, Chiles Shaffer N, Gonzalez-Freire M, Shardell MD, Zoli M, Studenski SA, Ferrucci L: Early body composition, but not body mass, is associated with future accelerated decline in muscle quality. J Cachexia Sarcopenia Muscle 2017, 8(3):490-499.

34. Bittner V, Weiner DH, Yusuf S, Rogers WJ, McIntyre KM, Bangdiwala SI, Kronenberg MW, Kostis JB, Kohn RM, Guillotte $\mathrm{M}$ et al: Prediction of mortality and morbidity with a 6-minute walk test in patients with left ventricular dysfunction. SOLVD Investigators. Jama 1993, 270(14):1702-1707.

35. Guralnik JM, Simonsick EM, Ferrucci L, Glynn RJ, Berkman LF, Blazer DG, Scherr PA, Wallace RB: A short physical performance battery assessing lower extremity function: association with self-reported disability and prediction of mortality and nursing home admission. J Gerontol 1994, 49(2):M85-94.

36. Shumway-Cook A, Brauer S, Woollacott M: Predicting the probability for falls in community-dwelling older adults using the Timed Up \& Go Test. Phys Ther 2000, 80(9):896-903.

37. Smith E, Walsh L, Doyle J, Greene B, Blake C: Effect of a dual task on quantitative Timed Up and Go performance in community-dwelling older adults: A preliminary study. Geriatr Gerontol Int 2017, 17(8):1176-1182.
38. da Silva RA, Bilodeau M, Parreira RB, Teixeira DC, Amorim CF: Age-related differences in time-limit performance and force platform-based balance measures during one-leg stance. J Electromyogr Kinesiol 2013, 23(3):634-639.

39. Lamb SE, Jorstad-Stein EC, Hauer K, Becker C: Development of a common outcome data set for fall injury prevention trials: the Prevention of Falls Network Europe consensus. J Am Geriatr Soc 2005, 53(9):1618-1622.

40. Camargos FFO, Dias RC, Dias JMD, Freire MTF: Cross-cultural adaptation and evaluation of the psychometric properties of the Falls Efficacy Scale - International Among Elderly Brazilians (FES-IBRAZIL). Brazilian Journal of Physical Therapy 2010, 14:237-243.

41. Malachias MVB, Gomes MAM, Nobre F, Alessi A, Feitosa AD, Coelho EB: 7th Brazilian Guideline of Arterial Hypertension: Chapter 2 - Diagnosis and Classification. Arq Bras Cardiol 2016, 107(3 Suppl 3):7-13.

42. Heart rate variability. Standards of measurement, physiological interpretation, and clinical use. Task Force of the European Society of Cardiology and the North American Society of Pacing and Electrophysiology. Eur Heart J 1996, 17(3):354-381.

43. Almeida OP, Almeida SA: Reliability of the Brazilian version of the geriatric depression scale (GDS) short form. Arquivos de Neuro-Psiquiatria 1999, 57:421-426.

44. Behm D, Colado JC: The effectiveness of resistance training using unstable surfaces and devices for rehabilitation. Int J Sports Phys Ther 2012, 7(2):226-241.

45. Behm DG, Colado JC, Colado JC: Instability resistance training across the exercise continuum. Sports Health 2013, 5(6):500-503.

\section{Corresponding author}

Bruno Remígio Cavalcante

Associated Graduate Program in Physical Education UPE/UFPB

University of Pernambuco - Campus Petrolina; BR $203 \mathrm{Km} 02 \mathrm{~s} / \mathrm{n}-$ Cidade Universitária - Vila Eduardo - CEP: 56328-903; Petrolina - PE; Brazil.

Email: br_13remigio@hotmail.com

Manuscript received on January 15, 2019

Manuscript accepted on February 20, 2019

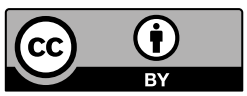

Motriz. The Journal of Physical Education. UNESP. Rio Claro, SP, Brazil - eISSN: 1980-6574 - under a license Creative Commons - Version 4.0 